\title{
Dynamics of nonlinear diatomic lattices
}

\author{
Michal Fečkan
}




\title{
DYNAMICS OF NONLINEAR DIATOMIC LATTICES
}

\author{
MICHAL FEČKAN
}

[Received: June 10, 2003 and, in revised form, June 22, 2003]

\begin{abstract}
A model of nonlinear diatomic lattice is studied. We suppose small damping, small forcing and weak coupling between the lattices. We show the existence of breathers for undamped and unforced cases. The existence of chaos is shown for damped and forced cases. For lattices with nonsmall parameters, the existence of travelling waves is discussed.
\end{abstract}

Mathematics Subject Classification: Primary 34C25; Secondary 34C37, 37K60, 37L60

Keywords: Diatomic lattices, bifurcations, breathers, chaos

\section{Introduction}

Let us consider a model of two one-dimensional interacting sublattices of harmonically coupled protons and heavy ions $[11,12,15,16]$. It represents the Bernal-Flower filaments in ice or more complex biological macromolecules in membranes, in which only the degrees of freedom that contribute predominantly to proton mobility have been conserved. In these systems, each proton lies between a pair of 'oxygens.' The proton part of the Hamiltonian is

$$
H_{p}=\sum_{n} \frac{1}{2} m \dot{u}_{n}^{2}+U\left(u_{n}\right)+\frac{1}{2} k_{1}\left(u_{n+1}-u_{n}\right)^{2},
$$

where $u_{n}$ denotes the displacement of the $n$th proton with respect to the center of the oxygen pair and $k_{1}$ is the coupling between neighboring protons. Furthermore,

$$
U(u)=\xi_{0}\left(1-u^{2} / d_{0}^{2}\right)^{2}
$$

is the double-well potential with the potential barrier $\xi_{0}$, and $2 d_{0}$ is the distance between its two minima. Finally, $m$ is the mass of protons.

Similarly, the oxygen part of the Hamiltonian is

$$
H_{O}=\sum_{n} \frac{1}{2} M \dot{\varrho}_{n}^{2}+\frac{1}{2} M \Omega_{0}^{2} \varrho_{n}^{2}+\frac{1}{2} K_{1}\left(\varrho_{n+1}-\varrho_{n}\right)^{2},
$$

This work was supported by the grant GA-SAV 2/1140/21. 
where $\varrho_{n}$ is the displacement between two oxygens, $M$ is the mass of oxygens, $\Omega_{0}$ is the frequency of the optical mode and $K_{1}$ is the harmonic coupling between neighboring oxygens.

The last part in the Hamiltonian of the model arises from the dynamic interaction between two sublattices and it is given by

$$
H_{\text {int }}=\sum_{n} \chi \varrho_{n}\left(u_{n}^{2}-d_{0}^{2}\right),
$$

where $\chi$ measures the strength of the coupling. The Hamiltonian of the model is the sum of these three contributions

$$
H=H_{p}+H_{O}+H_{\text {int }} .
$$

We are also interested in the influence of external field and damping. For the model studied here, since a spatially homogeneous field is not coupled to the optical motion $\varrho_{n}$ of the oxygens, a force term has to be considered only in the equation of motion of the protons.

Summarizing, we consider in this note the following coupled infinite chain of oscillators

$$
\begin{gathered}
\ddot{u}_{n}+\Gamma_{1} \dot{u}_{n}=\frac{k_{1}}{m}\left(u_{n+1}-2 u_{n}+u_{n-1}\right)+\frac{4 \xi_{0}}{m d_{0}^{2}} u_{n}\left(1-\frac{u_{n}^{2}}{d_{0}^{2}}\right) \\
-2 \frac{\chi}{m} \varrho_{n} u_{n}+\frac{F}{m}, \\
\ddot{\varrho}_{n}+\Gamma_{2} \dot{\varrho}_{n}=\frac{K_{1}}{M}\left(\varrho_{n+1}-2 \varrho_{n}+\varrho_{n-1}\right)-\Omega_{0}^{2} \varrho_{n}-\frac{\chi}{M}\left(u_{n}^{2}-d_{0}^{2}\right),
\end{gathered}
$$

where $F$ is the external force on the protons and $\Gamma_{1}, \Gamma_{2}$ are the damping coefficients for the proton and oxygen motions.

The contents of the paper is as follows. In Section 2, we study the weakly coupled undamped and unforced chain (1.1), i. e., with $\Gamma_{1}=\Gamma_{2}=0, F=0$ and the coupling parameters are small. We show under certain nonresonance conditions the existence of time periodic spatially localized solutions of (1.1), the so-called breathers $[5,9,14]$. Section 3 is devoted to the case when (1.1) is weakly forced and weakly damped with again weak coupling. By using a Melnikov method [10], we show under certain conditions the existence of spatially localized Smale horseshoes in (1.1), i. e., spatially localized chaos in (1.1). The localization of the Smale horseshoes is not exponential as for the breathers. Section 4 deals with (1.1) when the involved parameters are not small. The existence of travelling waves for (1.1) and also for its continuum limit is discussed.

\section{WEAKLY COUPLED UNDAMPED AND UNFORCED LATTICES: THE EXISTENCE OF BREATHERS}

We assume in this section that $\Gamma_{1}=\Gamma_{2}=F=0, k_{1} / m=\varepsilon \mu_{1}, K_{1} / M=\varepsilon \mu_{2}$, $-2 \chi / m=\varepsilon \mu_{3},-\chi / M=\varepsilon \mu_{4}$ for a small parameter $\varepsilon$ and constants $\mu_{i}, i=1,2,3,4$. 
Hence (1.1) has the form

$$
\begin{gathered}
\ddot{u}_{n}=\varepsilon \mu_{1}\left(u_{n+1}-2 u_{n}+u_{n-1}\right)+a^{2} u_{n}\left(d_{0}^{2}-u_{n}^{2}\right)+\varepsilon \mu_{3} \varrho_{n} u_{n}, \\
\ddot{\varrho}_{n}=\varepsilon \mu_{2}\left(\varrho_{n+1}-2 \varrho_{n}+\varrho_{n-1}\right)-\Omega_{0}^{2} \varrho_{n}+\varepsilon \mu_{4}\left(u_{n}^{2}-d_{0}^{2}\right),
\end{gathered}
$$

where $a^{2}=\frac{4 \xi_{0}}{m d_{0}^{4}}$. Then for $\varepsilon=0$, we get from (2.1) the uncoupled system

$$
\ddot{u}_{n}=a^{2}\left(d_{0}^{2}-u_{n}^{2}\right) u_{n}, \quad \ddot{\varrho}_{n}=-\Omega_{0}^{2} \varrho_{n} .
$$

The equation

$$
\dot{u}=v, \quad \dot{v}=a^{2}\left(d_{0}^{2}-u^{2}\right) u
$$

has a hyperbolic equilibrium $u=v=0$ and centers $u= \pm d_{0}, v=0$ [17]. Furthermore, equation (2.2) has two symmetric periodic solutions $\left(\gamma_{\beta}(t), \dot{\gamma}_{\beta}(t)\right)$ and $\left(-\gamma_{\beta}(t),-\dot{\gamma}_{\beta}(t)\right)$ around $\left( \pm d_{0}, 0\right)$ with periods $\beta$ monotonically increasing from $\frac{\sqrt{2} \pi}{a d_{0}}$ to $+\infty$. They accumulate on two symmetric homoclinic solutions $(\gamma(t), \dot{\gamma}(t))$ and $(-\gamma(t),-\dot{\gamma}(t))$ for

$$
\gamma(t)=\sqrt{2} d_{0} \operatorname{sech} a d_{0} t .
$$

We assume that $\gamma_{\beta}(t)$ and $\gamma(t)$ are even functions. We are interested, in this section, in spatially localized time periodic solutions of (2.1) which are called breathers. We use the approach of $[5,9,14]$. For this reason, we take the exchange $u_{n} \leftrightarrow u_{n}+d_{0}$ in (2.1) to get

$$
\begin{aligned}
\ddot{u}_{n}+a^{2} u_{n}\left(u_{n}+d_{0}\right)\left(u_{n}+2 d_{0}\right) & \\
-\varepsilon \mu_{1}\left(u_{n+1}-2 u_{n}+u_{n-1}\right)-\varepsilon \mu_{3} \varrho_{n}\left(u_{n}+d_{0}\right) & =0, \\
\ddot{\varrho}_{n}+\Omega_{0}^{2} \varrho_{n}-\varepsilon \mu_{2}\left(\varrho_{n+1}-2 \varrho_{n}+\varrho_{n-1}\right)-\varepsilon \mu_{4} u_{n}\left(u_{n}+2 d_{0}\right) & =0 .
\end{aligned}
$$

Now we fix a constant $\tau>1$ and consider the Banach spaces

$$
\begin{aligned}
X_{i}=\left\{\left\{\left(u_{n}(t), \varrho_{n}(t)\right)\right\}_{n \in \mathbb{Z}}\right. & \in C^{i}(\mathbb{R}, \mathbb{R})^{2 \mathbb{Z}} \mid u_{n}, \varrho_{n} \text { are even, } \\
& \left.\beta \text {-periodic, and such that } \sup _{n} \tau^{|n|}\left(\left|u_{n}(\cdot)\right|_{i}+\left.\varrho_{n}(\cdot)\right|_{i}\right)<\infty\right\},
\end{aligned}
$$

where $i=0,2$ and $|\cdot|_{i}$ is the usual maximum norm on $C^{i}([0, \beta], \mathbb{R})$. The norm on $X_{i}$ for $x=\left\{\left(u_{n}(t), \varrho_{n}(t)\right)\right\}_{n \in \mathbb{Z}}$ is defined as $\|x\|=\sup _{n} \tau^{|n|}\left(\left|u_{n}(\cdot)\right|_{i}+\left|\varrho_{n}(\cdot)\right|_{i}\right)$. The left-hand side of (2.3) defines the mapping $F: X_{2} \times \mathbb{R} \rightarrow X_{0}$ with $\varepsilon \in \mathbb{R}$. Hence (2.3) has the form

$$
F(x, \varepsilon)=0 .
$$

We take $x_{0} \in X_{2}$ with $u_{n}=0$ for $n \neq 0, u_{0}=\gamma_{\beta}(t)-d_{0}$ and $\varrho_{n}=0$ for any $n$. Then $F\left(x_{0}, 0\right)=0$. Clearly $F \in C^{\infty}\left(X_{2} \times \mathbb{R}, X_{0}\right)$. We solve (2.4) by using the implicit 
function theorem. The linearization $D_{x} F\left(x_{0}, 0\right): X_{2} \rightarrow X_{0}$ has the form

$$
\begin{gathered}
\left(\ddot{u}_{n}+2 a^{2} d_{0}^{2} u_{n}, \ddot{\varrho}_{n}+\Omega_{0}^{2} \varrho_{n}\right), \quad n \neq 0, \\
\left(\ddot{u}_{0}+a^{2}\left(3 \gamma_{\beta}(t)^{2}-d_{0}^{2}\right) u_{0}, \ddot{\varrho}_{n}+\Omega_{0}^{2} \varrho_{n}\right) .
\end{gathered}
$$

The equation

$$
\ddot{v}+a^{2}\left(3 \gamma_{\beta}(t)^{2}-d_{0}^{2}\right) v=0
$$

has the solutions $v_{1}(t)=\dot{\gamma}_{\beta}(t)$ and $v_{2}(t)=D_{\beta} \gamma_{\beta}(t)$. Function $v_{1}(t)$ is odd and $\beta$ periodic, while function $v_{2}(t)$ is even and satisfies the relation $v_{2}(t+\beta)=v_{2}(t)-\dot{\gamma}_{\beta}(t+$ $\beta$ ). Since $\dot{\gamma}_{\beta}(t+\beta) \neq 0$, the function $v_{2}(t)$ is not $\beta$-periodic. We note that the existence of an even and $\beta$-periodic solution of the equation

$$
\ddot{v}+a^{2}\left(3 \gamma_{\beta}(t)^{2}-d_{0}^{2}\right) v=h(t)
$$

for an even and $\beta$-periodic continuous function $h(t)$, is equivalent to the existence of a solution of the following boundary value problem

$$
\begin{gathered}
\ddot{v}+a^{2}\left(3 \gamma_{\beta}(t)^{2}-d_{0}^{2}\right) v=h(t) \\
\dot{v}(0)=\dot{v}(\beta / 2)=0 .
\end{gathered}
$$

Since the homogeneous problem of (2.6) with $h(t)=0$ has the only zero solution, we get that (2.6) is uniquely solvable. Consequently, (2.5) has a unique even and $\beta$-periodic solution.

Furthermore, we can directly check that the equations

$$
\begin{aligned}
& \ddot{u}+2 a^{2} d_{0}^{2} u=h(t), \\
& \ddot{\varrho}+\Omega_{0}^{2} \varrho=h(t)
\end{aligned}
$$

have unique even and $\beta$-periodic solutions for any even and $\beta$-periodic continuous function $h(t)$ if the following nonresonance conditions hold

$$
2 \sqrt{2} \frac{\sqrt{\xi_{0}}}{\sqrt{m} d_{0}} \beta=\sqrt{2} a d_{0} \beta \neq 2 \pi k, \quad \Omega_{0} \beta \neq 2 \pi k
$$

for any $k \in \mathbb{N}$. Consequently, we see that $D_{x} F\left(x_{0}, 0\right): X_{2} \rightarrow X_{0}$ is continuously invertible if (2.7) hold. The implicit function theorem implies the following result.

Theorem 2.1. Let $\tau>1$ be given. If the conditions (2.7) hold, then the chain (2.1) has a $\beta$-periodic solution $\left\{\left(u_{n}(t), \varrho_{n}(t)\right)\right\}_{n \in \mathbb{Z}}$ for any $\varepsilon$ small such that

$$
\sup _{t, n}\left(\left|u_{n}(t)-d_{0}\right|+\left|\varrho_{n}(t)\right|\right) \tau^{|n|}<\infty .
$$

Moreover, the relation $\varrho_{n}(t)=O(\varepsilon)$ is true for any $n$. Furthermore, $u_{n}(t)=d_{0}+O(\varepsilon)$ for $n \neq 0$ and $u_{0}(t)=\gamma_{\beta}(t)+O(\varepsilon)$. 
Under assumptions of Theorem 2.1, we get more complicated dynamics of (2.1). Namely, if we start from $x_{0} \in X_{2}$ such that $\varrho_{n}=0$ for any $n, u_{n}=0$ for large $n$ while $u_{n}=\gamma_{\beta}(t)-d_{0}$ for several finite numbers of $n$. Then we get for any $\varepsilon$ small, under conditions (2.7), the existence of multi-site breathers. Furthermore, if we take an infinite number of excitations, i. e., $u_{n}=\gamma_{\beta}(t)-d_{0}$ for infinite numbers of $n$ in the above construction of $x_{0}$, then we can repeat the proof of Theorem 2.1 for $\tau=1$ to get $\beta$-periodic solutions of (2.1), which are not spatially localized. But they are near to $\left(\gamma_{\beta}(t), 0\right)$ in infinitely many modes $n$. Moreover, the same arguments hold when we consider $-d_{0}$ instead of $d_{0}$ in the above considerations, i. e., we take the exchange $u_{n} \leftrightarrow u_{n}-d_{0}$ in (2.1). Furthermore, if (2.7) hold for some $\beta_{0}$, then (2.7) hold also for any $\beta$ near to $\beta_{0}$ and Theorem 2.1 can be applied uniformly for such $\beta$ and $\varepsilon$ small. In particular, we get under assumptions of Theorem 2.1 in (2.1) for $\varepsilon$ small infinitely many 1-parametric families of breathers.

Finally, if we start from $x_{0}$ such that either $u_{n}=-d_{0}, \varrho_{n}=0$ or $u_{n}=\gamma_{\beta}(t)-d_{0}$ and $\varrho_{n}=0$, then we consider $F(x, \varepsilon): X_{2} \times \mathbb{R} \rightarrow X_{0}$ for $\tau=1$, and we can repeat the proof of Theorem 2.1 to get the next result.

Theorem 2.2. If the conditions

$$
\Omega_{0} \beta \neq 2 \pi k \quad \text { for any } \quad k \in \mathbb{N}
$$

hold, then for any $E=\left\{e_{n}\right\}_{n \in \mathbb{Z}} \in\{0,1\}^{\mathbb{Z}}$ the chain (2.1) has a $\beta$-periodic, in general not localized spatially, solution $\left\{\left(u_{n, E}(t), \varrho_{n, E}(t)\right)\right\}_{n \in \mathbb{Z}}$ for any $\varepsilon$ small such that $\varrho_{n, E}(t)=O(\varepsilon)$ for any $n$, and $u_{n, E}(t)=O(\varepsilon)$ for $n$ with $e_{n}=1$ and $u_{n, E}(t)=\gamma_{\beta}(t)+O(\varepsilon)$ for $n$ with $e_{n}=0$.

We also note that the above results can be extended to the case when for (2.3) the element $x_{0}$ is such that $\varrho_{n}=0$ for any $n$, and either $u_{n}(t)=\gamma_{\beta}(t)-d_{0}$ or $u_{n}=0$ or $u_{n}=-d_{0}$ or $u_{n}=-2 d_{0}$. Summarizing we see that the dynamics of (2.1) is rather complicated for $\varepsilon \neq 0$ small.

\section{GeNeral WEAKLY COUPLED LATTICES: THE EXISTENCE OF CHAOS}

We assume in this section that $\Gamma_{1}=\varepsilon \delta_{1}, \Gamma_{2}=\varepsilon \delta_{2}, F=\varepsilon f(t), k_{1} / m=\varepsilon \mu_{1}$, $K_{1} / M=\varepsilon \mu_{2},-2 \chi / m=\varepsilon \mu_{3},-\chi / M=\varepsilon \mu_{4}$ for a small parameter $\varepsilon$, constants $\delta_{1} \geq 0$, $\delta_{2}>0, \mu_{i}, i=1,2,3,4$, and a $C^{1}$-smooth $T$-periodic function $f(t)$. Hence (1.1) has the form

$$
\begin{aligned}
\ddot{u}_{n}+\varepsilon \delta_{1} \dot{u}_{n}+a^{2} u_{n}\left(u_{n}^{2}-d_{0}^{2}\right)=\varepsilon \mu_{1}\left(u_{n+1}-\right. & \left.2 u_{n}+u_{n-1}\right) \\
& +\varepsilon \mu_{3} \varrho_{n} u_{n}+\varepsilon f(t), \\
\ddot{\varrho}_{n}+\varepsilon \delta_{2} \dot{\varrho}_{n}+\Omega_{0}^{2} \varrho_{n}=\varepsilon \mu_{2}\left(\varrho_{n+1}-\right. & \left.2 \varrho_{n}+\varrho_{n-1}\right) \\
& +\varepsilon \mu_{4}\left(u_{n}^{2}-d_{0}^{2}\right) .
\end{aligned}
$$


We first consider the system

$$
\begin{gathered}
\ddot{u}+\varepsilon \delta_{1} \dot{u}+a^{2} u\left(u^{2}-d_{0}^{2}\right)=\varepsilon \mu_{3} \varrho u+\varepsilon f(t), \\
\ddot{\varrho}+\varepsilon \delta_{2} \dot{\varrho}+\Omega_{0}^{2} \varrho=\varepsilon \mu_{4}\left(u^{2}-d_{0}^{2}\right) .
\end{gathered}
$$

We make the change of variable $\varrho \leftrightarrow \varrho-\frac{\varepsilon \mu_{4} d_{0}^{2}}{\Omega_{0}^{2}}$ in (3.2) to get

$$
\begin{gathered}
\ddot{u}+\varepsilon \delta_{1} \dot{u}+a^{2} u\left(u^{2}-d_{0}^{2}\right)=\varepsilon \mu_{3}\left(\varrho-\frac{\varepsilon \mu_{4} d_{0}^{2}}{\Omega_{0}^{2}}\right) u+\varepsilon f(t), \\
\ddot{\varrho}+\varepsilon \delta_{2} \dot{\varrho}+\Omega_{0}^{2} \varrho=\varepsilon \mu_{4} u^{2} .
\end{gathered}
$$

To study a small $T$-periodic solution of the above system, we take its equivalent form

$$
\begin{aligned}
\ddot{u}+\varepsilon \delta_{1} \dot{u} & +a^{2} u\left(u^{2}-d_{0}^{2}\right) \\
= & \varepsilon \mu_{3}\left(\frac{\varepsilon \mu_{4}}{\Omega_{\varepsilon}} \int_{-\infty}^{t} \mathrm{e}^{-\varepsilon \delta_{2}(t-s) / 2} \sin \Omega_{\varepsilon}(t-s) u^{2}(s) d s-\frac{\varepsilon \mu_{4} d_{0}^{2}}{\Omega_{0}^{2}}\right) u+\varepsilon f(t),
\end{aligned}
$$

where $\Omega_{\varepsilon}=\sqrt{\Omega_{0}^{2}-\frac{\varepsilon^{2} \delta_{2}^{2}}{4}}$ and $0<\varepsilon<2 \Omega_{0} / \delta_{2}$. Now it is not difficult to prove in (3.3) by using the implicit function theorem the existence of a unique small $T$-periodic solution $u_{\varepsilon}(t)=O(\varepsilon), \varrho_{\varepsilon}(t)=O(\varepsilon)$ of (3.2). Then we make in (3.1) the change of variables $u_{n} \leftrightarrow u_{n}+u_{\varepsilon}, \varrho_{n} \leftrightarrow \varrho_{n}+\varrho_{\varepsilon}$ to get the chain

$$
\begin{aligned}
\dot{u}_{n} & =v_{n}, \\
\dot{v}_{n}+\varepsilon \delta_{1} v_{n} & -a^{2} u_{n} d_{0}^{2}+a^{2} u_{n}^{3}+3 a^{2} u_{n}^{2} u_{\varepsilon}+3 a^{2} u_{n} u_{\varepsilon}^{2} \\
& =\varepsilon \mu_{1}\left(u_{n+1}-2 u_{n}+u_{n-1}\right)+\varepsilon \mu_{3}\left(\varrho_{n} u_{n}+\varrho_{n} u_{\varepsilon}+\varrho_{\varepsilon} u_{n}\right), \\
\dot{\varrho}_{n} & =\psi_{n}, \\
\dot{\psi}_{n}+\varepsilon \delta_{2} \psi_{n}+\Omega_{0}^{2} \varrho_{n} & =\varepsilon \mu_{2}\left(\varrho_{n+1}-2 \varrho_{n}+\varrho_{n-1}\right)+\varepsilon \mu_{4}\left(u_{n}^{2}+2 u_{\varepsilon} u_{n}\right) .
\end{aligned}
$$

We consider (3.4) as an ordinary differential equation in the Hilbert space

$$
H:=\left\{z=\left\{\left(u_{n}, v_{n}, \varrho_{n}, \psi_{n}\right)\right\}_{n \in \mathbb{Z}} \mid \sum_{n \in \mathbb{Z}}\left(u_{n}^{2}+v_{n}^{2}+\varrho_{n}^{2}+\psi_{n}^{2}\right)<\infty\right\}
$$

with the norm $\|z\|=\sqrt{\sum_{n \in \mathbb{Z}}\left(u_{n}^{2}+v_{n}^{2}+\varrho_{n}^{2}+\psi_{n}^{2}\right)}$. The inhomogeneous linearization of (3.4) at $z=0$ has the form

$$
\begin{aligned}
\dot{u}_{n} & =v_{n}+h_{n 1}(t) \\
\dot{v}_{n}+\varepsilon \delta_{1} v_{n} & +u_{n}\left(3 a^{2} u_{\varepsilon}^{2}-a^{2} d_{0}^{2}-\varepsilon \mu_{3} \varrho_{\varepsilon}\right) \\
& -\varepsilon \mu_{1}\left(u_{n+1}-2 u_{n}+u_{n-1}\right)-\varepsilon \mu_{3} \varrho_{n} u_{\varepsilon}=h_{n 2}(t), \\
\dot{\varrho}_{n} & =\psi_{n}+g_{n 1}(t), \\
\dot{\psi}_{n}+\varepsilon \delta_{2} \psi_{n} & +\Omega_{0}^{2} \varrho_{n}-\varepsilon \mu_{2}\left(\varrho_{n+1}-2 \varrho_{n}+\varrho_{n-1}\right) \\
& -2 \varepsilon \mu_{4} u_{\varepsilon} u_{n}=g_{n 2}(t)
\end{aligned}
$$


with $w(t)=\left\{\left(h_{n 1}(t), h_{n 2}(t), g_{n 1}(t), g_{n 2}(t)\right)\right\}_{n \in \mathbb{Z}} \in C_{b}(\mathbb{R}, H)$, where $C_{b}(\mathbb{R}, H)$ is the Banach space of all bounded continuous functions from $\mathbb{R}$ to $H$ with the norm $|w|=$ $\sup _{\mathbb{R}}\|w(t)\|$. We look for a solution $z \in C_{b}(\mathbb{R}, H)$ of (3.4) for $\varepsilon>0$ small. For this reason, we consider the Hilbert spaces $H_{2}:=H_{1} \times H_{1}$ and

$$
H_{1}:=\left\{\left\{u_{n}\right\}_{n \in \mathbb{Z}} \mid \sum_{n \in \mathbb{Z}} u_{n}^{2}<\infty\right\}
$$

with the corresponding standard norms. We first study the equation

$$
\begin{gathered}
\dot{\varrho}=\psi+g_{1} \\
\dot{\psi}+\varepsilon \delta_{2} \psi+A_{\varepsilon} \varrho=g_{2}
\end{gathered}
$$

on $H_{2}$ for $\left(g_{1}, g_{2}\right) \in C_{b}\left(\mathbb{R}, H_{2}\right)$. Here,

$$
A_{\varepsilon} \varrho=\left\{\Omega_{0}^{2} \varrho_{n}-\varepsilon \mu_{2}\left(\varrho_{n+1}-2 \varrho_{n}+\varrho_{n-1}\right)\right\}_{n \in \mathbb{Z}} .
$$

Clearly $A_{\varepsilon}: H_{1} \rightarrow H_{1}$ is symmetric and positive definite for $\varepsilon$ small. Then for any small $\varepsilon$, there is a symmetric and positive definite $B_{\varepsilon}: H_{1} \rightarrow H_{1}$ such that

$$
B_{\varepsilon}^{2}=A_{\varepsilon}-\frac{\varepsilon^{2} \delta_{2}^{2}}{4} I
$$

We take the operators $\cos B_{\varepsilon} t$ and $\sin B_{\varepsilon} t$ from $H_{1}$ to $H_{1}$. For any $\varrho \in H_{1}$, we consider the function

$$
\phi(t):=\left|\cos B_{\varepsilon} t \varrho\right|^{2}+\left|\sin B_{\varepsilon} t \varrho\right|^{2} .
$$

Then we have

$$
\dot{\phi}(t)=-2\left\langle\cos B_{\varepsilon} t \varrho, B_{\varepsilon} \sin B_{\varepsilon} t \varrho\right\rangle+2\left\langle\sin B_{\varepsilon} t \varrho, B_{\varepsilon} \cos B_{\varepsilon} t \varrho\right\rangle=0 .
$$

Hence

$$
\left|\cos B_{\varepsilon} t \varrho\right|^{2}+\left|\sin B_{\varepsilon} t \varrho\right|^{2}=\varrho,
$$

and then $\left\|\cos B_{\varepsilon} t\right\| \leq 1$ and $\left\|\sin B_{\varepsilon} t\right\| \leq 1$.

Now, the equation

$$
\begin{gathered}
\dot{\varrho}=\psi \\
\dot{\psi}+\varepsilon \delta_{2} \psi+A_{\varepsilon} \varrho=0
\end{gathered}
$$

has the form $\ddot{\varrho}+\varepsilon \delta_{2} \dot{\varrho}+A_{\varepsilon} \varrho=0$ which has the general solution

$$
\mathrm{e}^{-\varepsilon \delta_{2} t / 2}\left[\cos B_{\varepsilon} t \varrho_{1}+\sin B_{\varepsilon} t \varrho_{2}\right]
$$

for $\varrho_{1,2} \in H_{1}$. Consequently, the fundamental solution of (3.7) has the form

$$
V_{\varepsilon}(t)=\mathrm{e}^{-\varepsilon \delta_{2} t / 2} W_{\varepsilon}(t)
$$

with uniformly bounded $W_{\varepsilon}(t)$ for $\varepsilon>0$ small. Thus, the only bounded solution of (3.6) has the form

$$
(\varrho(t), \psi(t))=\int_{-\infty}^{t} \mathrm{e}^{-\varepsilon \delta_{2}(t-s) / 2} W_{\varepsilon}(t-s)\left(g_{1}(s), g_{2}(s) d s .\right.
$$


Hence

$$
|(\varrho, \psi)| \leq K_{1}\left|\left(g_{1}, g_{2}\right)\right| / \varepsilon
$$

for a constant $K_{1}>0$ independent of $\varepsilon>0$ small.

Furthermore, it is not difficult to see that the linear system

$$
\begin{gathered}
\dot{u}_{n}=v_{n}+h_{n 1}(t) \\
\dot{v}_{n}+\varepsilon \delta v_{n}-a^{2} d_{0}^{2} u_{n}=h_{n 2}(t)
\end{gathered}
$$

has a unique solution $\left\{\left(u_{n}(t), v_{n}(t)\right)\right\}_{n \in \mathbb{Z}} \in C_{b}\left(\mathbb{R}, H_{2}\right)$ such that

$$
\left|\left\{\left(u_{n}(t), v_{n}(t)\right)\right\}_{n \in \mathbb{Z}}\right| \leq K_{2}\left|\left\{\left(h_{n 1}(t), h_{n 2}(t)\right)\right\}_{n \in \mathbb{Z}}\right|
$$

for a constant $K_{2}>0$ independent of $\varepsilon>0$ small.

Now we return to (3.5). Summarizing the above arguments, we see, by using the Banach contraction mapping principle for $\varepsilon>0$ small, that (3.5) has for any $w(t) \in C_{b}(\mathbb{R}, H)$ a unique solution $z \in C_{b}(\mathbb{R}, H)$ such that $|z| \leq K_{3}|w| / \varepsilon$ for a constant $K_{3}>0$ independent of $\varepsilon>0$ small. Since the system (3.5) is $T$-periodic, then we get from the proof of Theorem 2.1 of [4, p. 288] that (3.5) has an exponential dichotomy on $\mathbb{R}$ in the space $H$ for any $\varepsilon>0$ sufficiently small. Consequently, we get the next result.

Theorem 3.1. The T-periodic solution $u_{n}(t)=u_{\varepsilon}(t), \varrho_{n}(t)=\varrho_{\varepsilon}(t) \forall n \in \mathbb{Z}$ of $(3.1)$ is hyperbolic in $H$ for any $\varepsilon>0$ sufficiently small, $i$. e., the zero equilibrium of (3.4) in $H$ is hyperbolic.

Now we look for more complicated solutions of (3.1). For this reason, we shift in (3.4) the time $t \leftrightarrow t+\alpha$ to get the system

$$
\begin{gathered}
\dot{u}_{n}=v_{n}, \\
\dot{v}_{n}+\varepsilon \delta_{1} v_{n}-a^{2} u_{n} d_{0}^{2}+a^{2} u_{n}^{3}+3 a^{2} u_{n}^{2} u_{\varepsilon}(t+\alpha) \\
+3 a^{2} u_{n} u_{\varepsilon}^{2}(t+\alpha)=\varepsilon \mu_{1}\left(u_{n+1}-2 u_{n}+u_{n-1}\right) \\
+\varepsilon \mu_{3}\left(\varrho_{n} u_{n}+\varrho_{n} u_{\varepsilon}(t+\alpha)+\varrho_{\varepsilon}(t+\alpha) u_{n}\right), \\
\dot{\varrho}_{n}=\psi_{n}, \\
\dot{\psi}_{n}+\varepsilon \delta_{2} \psi_{n}+\Omega_{0}^{2} \varrho_{n}=\varepsilon \mu_{2}\left(\varrho_{n+1}-2 \varrho_{n}+\varrho_{n-1}\right)+\varepsilon \mu_{4}\left(u_{n}^{2}+2 u_{\varepsilon}(t+\alpha) u_{n}\right) .
\end{gathered}
$$

We look for a solution of (3.9) for $\varepsilon>0$ small such that $u_{n} \sim 0, v_{n} \sim 0$ for $n \neq 0$ and $u_{0} \sim \gamma, v_{0} \sim \dot{\gamma}$.

Let $\left(\varrho_{0}, \psi_{0}\right)=\left\{\left(\varrho_{n}^{0}, \psi_{n}^{0}\right)\right\}_{n \in \mathbb{Z}}$ be the unique bounded solution of (3.6) for $g_{1}=0$ and $g_{2}=\left\{g_{n 2}\right\}_{n \in \mathbb{Z}}$ with $g_{n 2}=0$ for $n \neq 0$ and

$$
g_{02}=\varepsilon \mu_{4}\left(\gamma^{2}+2 u_{\varepsilon}(t+\alpha) \gamma\right)
$$

Let us put $u_{n}^{0}=v_{n}^{0}=0$ for $n \neq 0$ and $u_{0}^{0}=\gamma, v_{0}^{0}=\dot{\gamma}$. Now we make in (3.9) the change of variables $u_{n} \leftrightarrow u_{n}+u_{n}^{0}, v_{n} \leftrightarrow v_{n}+v_{n}^{0}, \varrho_{n} \leftrightarrow \varrho_{n}+\varrho_{n}^{0}, \psi_{n} \leftrightarrow \psi_{n}+\psi_{n}^{0}$ to get, 
for $n \neq 0$, the system

$$
\begin{gathered}
\dot{u}_{n}=v_{n}, \\
\dot{v}_{n}+\varepsilon \delta_{1} v_{n}-a^{2} u_{n} d_{0}^{2}+a^{2} u_{n}^{3}+ \\
=8 a^{2} u_{n}^{2} u_{\varepsilon}(t+\alpha)+3 a^{2} u_{n} u_{\varepsilon}^{2}(t+\alpha) \\
=\varepsilon \mu_{1}\left(u_{n+1}+u_{n+1}^{0}-2 u_{n}+u_{n-1}+u_{n-1}^{0}\right) \\
+\varepsilon \mu_{3}\left(\left(\varrho_{n}+\varrho_{n}^{0}\right) u_{n}+\left(\varrho_{n}+\varrho_{n}^{0}\right) u_{\varepsilon}(t+\alpha)\right. \\
\left.+\varrho_{\varepsilon}(t+\alpha) u_{n}\right), \\
\dot{\varrho}_{n}=\psi_{n}, \\
\dot{\psi}_{n}+\varepsilon \delta_{2} \psi_{n}+\Omega_{0}^{2} \varrho_{n}=\varepsilon \mu_{2}\left(\varrho_{n+1}-2 \varrho_{n}+\varrho_{n-1}\right) \\
+\varepsilon \mu_{4}\left(u_{n}^{2}+2 u_{\varepsilon}(t+\alpha) u_{n}\right) .
\end{gathered}
$$

For the mode $n=0$, we first note that the system

$$
\begin{gathered}
\dot{u}_{0}=v_{0} \\
\dot{v}_{0}+a^{2}\left(3 \gamma^{2}-d_{0}^{2}\right) u_{0}=h(t)
\end{gathered}
$$

for $h(t) \in C_{b}(\mathbb{R}, \mathbb{R})$ has a solution $\left(u_{0}, v_{0}\right) \in C_{b}\left(\mathbb{R}, \mathbb{R}^{2}\right)$ (see [10]) if and only if

$$
\int_{-\infty}^{\infty} h(t) \dot{\gamma}(t) d t=0
$$

and such a solution is unique if $\int_{-\infty}^{\infty} u_{0}(t) \dot{\gamma}(t) d t=0$. Consequently, for the mode $n=0$, we get from (3.9) the equations

$$
\begin{gathered}
\dot{u}_{0}=v_{0} \\
\dot{v}_{0}+a^{2}\left(3 \gamma^{2}-d_{0}^{2}\right) u_{0}=h(t)-\dot{\gamma}(t) \int_{-\infty}^{\infty} h(t) \dot{\gamma}(t) d t / \int_{-\infty}^{\infty} \dot{\gamma}(t)^{2} d t \\
\int_{-\infty}^{\infty} u_{0}(t) \dot{\gamma}(t) d t=0 \\
\dot{\varrho}_{0}=\psi_{0} \\
\dot{\psi}_{0}+\varepsilon \delta_{2} \psi_{0}+\Omega_{0}^{2} \varrho_{0}=\varepsilon \mu_{2}\left(\varrho_{1}-2 \varrho_{0}+\varrho_{-1}\right) \\
+\varepsilon \mu_{4}\left(u_{0}^{2}+2 u_{0} \gamma+2 u_{\varepsilon}(t+\alpha) u_{0}\right),
\end{gathered}
$$

and

$$
\int_{-\infty}^{\infty} h(t) \dot{\gamma}(t) d t=0
$$


for

$$
\begin{gathered}
h(t)=-a^{2}\left(u_{0}^{3}+3 u_{0}^{2} \gamma\right)-\varepsilon \delta_{1} \dot{\gamma}-3 a^{2}\left(u_{0}+\gamma\right)^{2} u_{\varepsilon}(t+\alpha)-\varepsilon \delta_{1} v_{0} \\
-3 a^{2}\left(u_{0}+\gamma\right) u_{\varepsilon}^{2}(t+\alpha)+\varepsilon \mu_{1}\left(u_{1}-2\left(u_{0}+\gamma\right)+u_{-1}\right) \\
+\varepsilon \mu_{3}\left(\left(\varrho_{0}+\varrho_{0}^{0}\right)\left(u_{0}+\gamma\right)+\left(\varrho_{0}+\varrho_{0}^{0}\right) u_{\varepsilon}(t+\alpha)\right. \\
\left.+\varrho_{\varepsilon}(t+\alpha)\left(u_{0}+\gamma\right)\right) .
\end{gathered}
$$

Now for $\varepsilon>0$ small, we can solve (3.10) and (3.11) to get the solution

$$
z=\left\{\left(u_{n}(t), v_{n}(t), \varrho_{n}(t), \psi_{n}(t)\right)\right\}_{n \in \mathbb{Z}} \in C_{b}(\mathbb{R}, H)
$$

such that $z=O(\varepsilon)$. Then we put this $z$ into (3.13) to get the function $h_{\varepsilon, \alpha} \in C_{b}(\mathbb{R}, \mathbb{R})$. We note $h_{\varepsilon, \alpha}(t)=O(\varepsilon)$ uniformly for $\varepsilon>0$ small and $\alpha, t \in \mathbb{R}$. Clearly $h_{\varepsilon, \alpha}(t)$ is $T$-periodic in $\alpha$. Then from (3.12) we get the bifurcation equation

$$
Q(\varepsilon, \alpha):=\frac{1}{\varepsilon} \int_{-\infty}^{\infty} h_{\varepsilon, \alpha}(t) \dot{\gamma}(t) d t=0 .
$$

We need to study the limit of $h_{\varepsilon, \alpha}(t)$ as $t \rightarrow 0$. If we put

$$
\lim _{\varepsilon \rightarrow 0} u_{\varepsilon}(t) / \varepsilon=w(t), \quad \lim _{\varepsilon \rightarrow 0} \varrho_{\varepsilon}(t) \varepsilon=\zeta(t),
$$

then from (3.2) we get

$$
\ddot{w}-a^{2} d_{0}^{2} w=f(t), \quad \ddot{\zeta}+\Omega_{0}^{2} \zeta=-\mu_{4} d_{0}^{2} .
$$

Hence $\zeta=-\mu_{4} d_{0}^{2} / \Omega_{0}^{2}$ and

$$
w(t)=-\frac{1}{2 a d_{0}} \int_{-\infty}^{t} \mathrm{e}^{-a d_{0}(t-s)} f(s) d s-\frac{1}{2 a d_{0}} \int_{t}^{\infty} \mathrm{e}^{a d_{0}(t-s)} f(s) d s .
$$

It is clear that $w(t)$ is $T$-periodic. Furthermore, since $\gamma(t) \rightarrow 0$ as $t \rightarrow \pm \infty$ exponentially, from the formula over (3.8) of the bounded solution of (3.6) we see that $\lim _{\varepsilon \rightarrow 0}\left(\varrho_{0}, \psi_{0}\right) / \varepsilon=\left\{\left(\varrho_{0 n}, \psi_{0 n}\right)\right\}_{n \in \mathbb{Z}}$ with $\varrho_{0 n}=\psi_{0 n}=0$ for $n \neq 0$ and

$$
\ddot{\varrho}_{00}+\Omega_{0}^{2} \varrho_{00}=\mu_{4} \gamma(t)^{2} \text {, }
$$

i. e.,

$$
\varrho_{00}(t)=\frac{\mu_{4}}{\Omega_{0}} \int_{-\infty}^{t} \sin \Omega_{0}(t-s) \gamma(s)^{2} d s .
$$

Summarizing, from (3.13) we get

$$
\begin{aligned}
M(\alpha):=Q(0, \alpha)= & \int_{-\infty}^{\infty}\left[-\delta_{1} \dot{\gamma}(t)-3 a^{2} \gamma(t)^{2} w(t+\alpha)\right. \\
& \left.-2 \mu_{1} \gamma(t)\right] \dot{\gamma}(t) d t=-\frac{4}{3} \delta_{1} a d_{0}^{3}+a^{2} \int_{-\infty}^{\infty} \gamma(t)^{3} \dot{w}(t+\alpha) d t .
\end{aligned}
$$


Clearly, $M(\alpha)$ is $T$-periodic. We note that similarly we can prove that

$$
\lim _{\varepsilon \rightarrow 0} \frac{\partial}{\partial \alpha} Q(\varepsilon, \alpha) / \varepsilon=M^{\prime}(\alpha)
$$

uniformly for $\alpha \in \mathbb{R}$. Summarizing, we get the next result.

Theorem 3.2. Let $M$ be given by (3.16). If there is a simple zero $\alpha_{0}$ of $M$, i. e., $M\left(\alpha_{0}\right)=0$ and $M^{\prime}\left(\alpha_{0}\right) \neq 0$, then (3.1) has for any $\varepsilon>0$ small a bounded solution $z(t)$ with small $u_{n}, \varrho_{n}$ for $n \neq 0$ and $\left(u_{0}, \varrho_{0}\right)$ near $\left(\gamma\left(t-\alpha_{0}\right), 0\right)$.

Now, it is not difficult to prove, as in the finite-dimensional case [10], that

$$
\left(z(t)-\left\{\left(u_{\varepsilon}(t), \dot{u}_{\varepsilon}(t), \varrho_{\varepsilon}(t), \dot{\varrho}_{\varepsilon}(t)\right)\right\}_{n \in \mathbb{Z}}\right) \rightarrow 0
$$

exponentially fast as $t \rightarrow \pm \infty$ in $H$. Moreover, near $z(t)$, we can construct the Smale horseshoe. Consequently, we get in this case chaos in (3.1) with corresponding infinitely many periodic orbits with arbitrarily large periods. This Smale horseshoe of (3.1) is spatially localized but not exponentially as in breathers.

To be more concrete, we take

$$
f(t)=\Upsilon \cos \omega t
$$

for $\Upsilon>0$. Then (3.15) gives

$$
w(t)=-\frac{\Upsilon}{\omega^{2}+a^{2} d_{0}^{2}} \cos \omega t,
$$

and the formula (3.16) has now the form

$$
M(\alpha)=-\frac{4}{3} \delta_{1} a d_{0}^{3}+\frac{\omega \Upsilon \pi \sqrt{2}}{a} \operatorname{sech} \frac{\omega \pi}{2 a d_{0}} \sin \omega \alpha .
$$

Consequently, if

$$
4 \sqrt{2} \delta_{1} \sqrt{\xi_{0}} d_{0}<3 \sqrt{m} \omega \Upsilon \pi \operatorname{sech} \frac{\omega \pi}{2 a d_{0}},
$$

then $M(\alpha)$ has a simple zero, so then (3.1) is chaotic for any $\varepsilon>0$ small. We note that the inequality (3.17) gives sufficient conditions between the magnitude of the forcing $\Upsilon$ and the damping $\delta_{1}$ in order to get chaos in (3.1) for $\varepsilon>0$ small. So chaos is generated by the proton part of (3.1). If $\delta_{1}=0$, then (3.1) is always chaotic for $f(t)=\Upsilon \cos \omega t$. Furthermore, if $f(t)=0$, i. e., there is no forcing, then it is not difficult to prove that (3.1) has no nonconstant periodic solutions in space $H$.

Finally, we note that similarly we can study the case when more than one modes are excited. We do not carry out such computations here. 


\section{Concluding remarks}

In this note, we have studied weakly coupled diatomoc lattices presented by model (1.1). In the case where (1.1) is unforced and undamped, the existence of infinitely many time-periodic and spatially localized solutions (the so-called breathers) has been shown. For small damping and forcing, we have proved the existence of infinitely many spatially localized Smale horseshoes, i. e., the existence of chaos in (1.1). The localization of the Smale horseshoes is not exponential as is the case for breathers. The proofs of these results are based on the use of the implicit function theorem. This would allow us to establish profiles of these solutions by using analytic-numeric methods as in [7].

For general 'non-small' parameters involved in (1.1), the study of dynamics of (1.1) is rather difficult. The existence of travelling waves could give some answers. There are two possibilities to handle this problem.

The first one is to consider directly in (1.1) travelling wave solutions

$$
u_{n}(t)=\phi(v t-n), \quad \varrho_{n}(t)=\psi(v t-n)
$$

to get the system

$$
\begin{aligned}
v^{2} \ddot{\phi}(t)+\Gamma_{1} v \dot{\phi}(t)= & \frac{k_{1}}{m}(\phi(t+1)-2 \phi(t)+\phi(t-1))+\frac{4 \xi_{0}}{m d_{0}^{2}} \phi(t)\left(1-\frac{\phi^{2}(t)}{d_{0}^{2}}\right) \\
& -2 \frac{\chi}{m} \psi(t) \phi(t)+\frac{F}{m}, \\
v^{2} \ddot{\psi}(t)+\Gamma_{2} v \dot{\psi}(t)= & \frac{K_{1}}{M}(\psi(t+1)-2 \psi(t)+\psi(t-1)) \\
& -\Omega_{0}^{2} \psi(t)-\frac{\chi}{M}\left(\phi(t)-d_{0}^{2}\right),
\end{aligned}
$$

where now $F$ is a constant external force. Equations of types similar to (4.1) are studied in [6].

The second one is to take the continuum limit of (1.1) to obtain the partial differential equation

$$
\begin{gathered}
u_{t t}+\Gamma_{1} u_{t}=\frac{k_{1}}{m} b^{2} u_{x x}+\frac{4 \xi_{0}}{m d_{0}^{2}} u\left(1-\frac{u^{2}}{d_{0}^{2}}\right)-2 \frac{\chi}{m} \varrho u+\frac{F}{m}, \\
\varrho_{t t}+\Gamma_{2} \varrho_{t}=\frac{K_{1}}{M} b^{2} \varrho_{x x}-\Omega_{0}^{2} \varrho-\frac{\chi}{M}\left(u^{2}-d_{0}^{2}\right),
\end{gathered}
$$

where again $F$ is a constant external force, $x=b n$ is the continuum space variable and $b>0$ is the lattice spacing. The travelling wave solutions

$$
u(x, t)=\phi(v t-x), \quad v(x, t)=\psi(v t-x)
$$


of (4.2) satisfy the system

$$
\begin{gathered}
\ddot{\phi}\left(v^{2}-\frac{k_{1}}{m} b^{2}\right)+\Gamma_{1} v \dot{\phi}=\frac{4 \xi_{0}}{m d_{0}^{2}} \phi\left(1-\frac{\phi^{2}}{d_{0}^{2}}\right)-2 \frac{\chi}{m} \psi \phi+\frac{F}{m}, \\
\ddot{\psi}\left(v^{2}-\frac{K_{1}}{M} b^{2}\right)+\Gamma_{2} v \dot{\psi}=-\Omega_{0}^{2} \psi-\frac{\chi}{M}\left(\phi^{2}-d_{0}^{2}\right) .
\end{gathered}
$$

System (4.3) is numerically studied in $[11,16]$. System (4.1) was not yet investigated. But for certain values of parameters in (4.1-4.3), the bifurcation methods as in this paper can be applied to find analytically either periodic, homoclinic or heteroclinic solutions of (4.1-4.3).

For instance, if

$$
k_{1} / m=K_{1} / M \quad \text { and } \quad v^{2} \sim K_{1} b^{2} / M,
$$

then by using the theory of singularly perturbed ordinary differential equations [3], the dynamics of (4.3) is reduced to the system

$$
\begin{gathered}
\Gamma_{1} v \dot{\phi}=\frac{4 \xi_{0}}{m d_{0}^{2}} \phi\left(1-\frac{\phi^{2}}{d_{0}^{2}}\right)-2 \frac{\chi}{m} \psi \phi+\frac{F}{m}, \\
\Gamma_{2} v \dot{\psi}=-\Omega_{0}^{2} \psi-\frac{\chi}{M}\left(\phi^{2}-d_{0}^{2}\right)
\end{gathered}
$$

for

$$
v^{2}=K_{1} b^{2} / M .
$$

System (4.4) admits the Lyapunov function

$$
L(\phi, \psi)=\frac{\xi_{0}}{m d_{0}^{2}}\left(1-\frac{\phi^{2}}{d_{0}^{2}}\right)^{2}-\frac{\chi}{M} \psi\left(\phi^{2}-d_{0}^{2}\right)-\Omega_{0}^{2} \frac{\psi^{2}}{2}+\frac{F}{m} \phi .
$$

Hence the limit sets of (4.4) are its equilibria. In general they are hyperbolic. So for

$$
v^{2} \sim K_{1} b^{2} / M,
$$

they persist in (4.3) and consequently, on bounded sets, these hyperbolic equilibria are limit sets of (4.3).

Similarly, we can study the case

$$
v^{2} \sim K_{1} b^{2} / M \quad \text { when } \quad k_{1} / m \neq K_{1} / M .
$$

This was numerically studied in [11]. The reduced system is now

$$
\begin{gathered}
\ddot{\phi}\left(v^{2}-\frac{k_{1}}{m} b^{2}\right)+\Gamma_{1} v \dot{\phi}=\frac{4 \xi_{0}}{m d_{0}^{2}} \phi\left(1-\frac{\phi^{2}}{d_{0}^{2}}\right)-2 \frac{\chi}{m} \psi \phi+\frac{F}{m}, \\
\Gamma_{2} v \dot{\psi}=-\Omega_{0}^{2} \psi-\frac{\chi}{M}\left(\phi^{2}-d_{0}^{2}\right)
\end{gathered}
$$


for $v^{2}=K_{1} b^{2} / M$. According to [3], the dynamics of bounded solutions of (4.3) is now approximated by (4.5). To study bounded solutions of (4.5), we consider its equivalent form given by the inegro-differential equation

$$
\begin{aligned}
\ddot{\phi}\left(v^{2}-\frac{k_{1}}{m} b^{2}\right)+\Gamma_{1} v \dot{\phi}=\frac{4 \xi_{0}}{m d_{0}^{2}} \phi & \left(1-\frac{\phi^{2}}{d_{0}^{2}}\right) \\
& +\frac{2 \chi^{2}}{v M m \Gamma_{2}} \phi \int_{-\infty}^{t} \mathrm{e}^{-\frac{\Omega_{0}^{2}(t-s)}{\Gamma_{2} v}}\left(\phi(s)^{2}-d_{0}^{2}\right) d s+\frac{F}{m} .
\end{aligned}
$$

Equation (4.6) can be studied similarly to (3.3) but we do not investigate it any further in this note. We postpone this to another paper.

\section{REFERENCES}

[1] Aubry, S., Kopidakis, G., and Kadelburg, V.: Variational prooffor hard discrete breathers in some classes of Hamiltonian dynamical systems, Discr. Cont. Dyn. Syst. B, 1 (2001), 271-298.

[2] Bambusi, D. and Vella, D.: Quasiperiodic breathers in Hamiltonian lattices with symmetries, Discr. Cont. Dyn. Syst. B, 2 (2002), 389-399.

[3] Battelli, F. AND FečKAn, M.: Global center manifolds in singular systems, Nonlinear Differential Equations Applications, 3 (1996), 19-34.

[4] Daletskit, Yu. L. and Krein, M. G.: Stability of Differential Equations, Nauka, Moscow, 1970. (in Russian)

[5] FečKAn, M.: Blue sky catastrophes in weakly coupled chains of reversible oscillators, Disc. Cont. Dyn. Syst. B, 3 (2003), 193-200.

[6] FečKan, M. And Rothos, V.: Bifurcations of periodics from homoclinics in singular O.D.E.: Applications to discretizations of travelling waves of p.d.e., Comm. Pure Appl. Anal., 1 (2002), 475-483.

[7] Haskins, M. AND SPEIGHT, J. M.: Breather initial profiles in chains of weakly coupled unharmonic oscillators, Phys. Lett. A, 299 (2002), 549-557.

[8] Haskins, M. and Speight, J. M.: Breathers in the weakly coupled topological discrete sine-Gordon system, Nonlinearity, 11 (1998), 1651-1671.

[9] Mackay, R. S. and Aubry, S.: Proof of existence of breathers for time-reversible or Hamiltonian networks of weakly coupled oscillators, Nonlinearity 7, (1994), 1623-1643.

[10] Palmer, K. J.: Exponential dichotomies, the shadowing lemma and transversal homoclinic points, Dynamics Reported, 1 (1988), 265-306.

[11] Peyrard, M., Pnevmatikos, S., and Flytzanis, N.: Dynamics of two-component solitary waves in hydrogen-bounded chains, Phys. Rev. A, 36 (1987), 903-914.

[12] Pnevmatikos, S., Flytzanis, N., and Remoissenet, M.: Soliton dynamics of nonlinear diatomic lattices, Phys. Rev. B, 33 (1986), 2308-2321.

[13] Samoilenko, A. M. and Teplinskis, Yu. V.: Countable Systems of Differential Equations, Kiev, 1993. (in Russian)

[14] Sepulchre, J. A. and Mackay, R. S.: Localized oscillations in conservative or dissipative networks of weakly coupled autonomous oscillators, Nonlinearity, 10 (1997), 679-713.

[15] Tew, R. B. and Wattis, J. A. D.: Quasi-continuum approximations for travelling kinks in diatomic lattices, J. Phys. A: Math. Gen., 34 (2001), 7163-7180.

[16] Wattis, J. A. D.: Solitary waves in a diatomic lattice: analytic approximations for a wide range of speeds by quasi-continuum methods, Phys. Lett. A, 284 (2001), 16-22. 
[17] Wiggins, S.: Global Bifurcations and Chaos, Analytical Methods, Applied Mathematical Sciences, 73, Springer-Verlag, New York, Heidelberg, Berlin, 1988.

\section{Author's Address}

\section{Michal Fečkan:}

Department of Mathematical Analysis, Comenius University, Mlynská dolina, 84248 Bratislava, SLOVAKIA

Mathematical Institute, Slovak Academy of Sciences, Štefánikova 49, 81473 Bratislava, Slovakia

E-mail address: Michal.Feckan@fmph.uniba.sk 